\title{
Mechanical Science III
}

\author{
P. R. Lancaster \\ D. Mitchell
}


All rights reserved. No part of this publication may be reproduced or transmitted, in any form or by any means, without permission.

First published 1977 by

THE MACMILLAN PRESS LTD

London and Basingstoke

Associated companies in Delhi Dublin

Hong Kong Johannesburg Lagos Melbourne

New York Singapore and Tokyo

\section{British Library Cataloguing in Publication Data}

\section{Lancaster, Philip Roy}

Mechanical Science III. -(Macmillan technician series).

I. Mechanics, Applied

I. Title II. Mitchell, D III. Series

$620.1 \quad$ TA350

ISBN 978-1-349-03145-0 ISBN 978-1-349-03143-6 (eBook)

DOI 10.1007/978-1-349-03143-6

This book is sold subject to the standard conditions of the Net Book Agreement.

The paperback edition of this book is sold subject to the condition that it shall not, by way of trade or otherwise, be lent, resold, hired out, or otherwise circulated without the publisher's prior consent in any form of binding or cover other than that in which it is published and without a similar condition including this condition being imposed on the subsequent purchaser. 


\section{Contents}

\section{PART ONE DEFORMATION OF MATERIAIS}

\section{Stress, Strain and Elasticity}

1.1 Compound Bars

1.2 The Effect of Temperature Change

1.3 Engineering and Temperature Strain

1.4 Shear Stress, Shear Strain, Modulus of Rigidity

1.4.1 Shear stress

1.4.2 Shear strain

1.4.3 Shear modulus

1.5 Poisson's Ratio

1.6 Problems involving Poisson's Ratio

2 Bending of Beams due to Transverse Forces

2.1 Uses of Beams and Bars

2.2 Types of Beam in Common Use

2.2.1 Simply supported beam

2.2.2 Cantilever

2.2.3 Built-in beams

2.3 Loading of Beams

2.3.1 Beam loaded by forces acting in one plane

2.4 Bending Moment, Moment of Resistance and Shear Force

2.5 Stresses and Strains in Beam Material

2.6 Design of Beams

2.7 Examples

2.7.1 Bending-moment limitation

2.7.2 Calculation of bending stress

2.7.3 Stress limitation

2.7.4 Bending-moment diagrams

2.7.5 Calculation of beam dimensions

2.7.6 Maximum allowable bending moment

2.7.7 Design of a beam to fulfil stress and deflection limitations

\section{A. DEFORMATION OF MATERIALS}

1. Uses stress, strain, elasticity in problems

Solves from first principles problems involving composite bars under uni-axial loads only, at uniform temperature. Solves similar problems but including the effect of uniform temperature change.

States that total direct strain is the sum of the strain due to loading and temperature change.

Defines (a) shear stress, (b) shear strain and (c) modulus of rigidity (shear modulus) and solves associated problems.

Defines Poisson's ratio.

Applies the Poisson's ratio effect in stress-strain relationships to solve associated problems in two dimensions (excluding shear stress action).

2. Develops, and uses, the simple theory of bending of symmetrical beams

Identifies, with the aid of a sketch, the position of a neutral plane in a symmetrical beam under the influence of a bending force system.

Defines bending moment.

Shows, by use of the equations of equilibrium, that under equilibrium conditions (a) the neutral plane (axis) passes through the centroid of cross-section, (b) the bending stress is given by $\sigma=M y / I$

Solves problems, with section characteristics restricted to rectangular, circular or idealised I-section beams, involving: (a) maximum allowable stresses, (b) bending moments or (c) loading conditions.

Given a specified section modulus $(Z)$ uses standard section handbooks to select appropriate beams. 
2.7.8 Use of BS 4: Part 1: 1972

2.8 Problems

3 Torsion of Circular Prisms

3.1 Introduction and Assumptions

3.2 Relationship between Shear Strain, Shear Stress and Angle of Twist

3.3 Relationship between Applied Torque, Second Moment of Area and Angle of Twist

3.4 Examples

3.4.1 Shear-stress distribution in solid shaft

3.4.2 Weight ecomony of hollow shaft

3.4.3 Transmission of power

3.4.4 Stepped shaft

3.4.5 Shafts of more than one material

\section{PART TWO DYNAMICS}

4 Angular Motion

4.1 Equations of Motion

4.2 Kinematics of a Particle Moving in a Circle with

Constant Angular Acceleration

4.3 Rotation of a Rigid Body about a Pivot

4.3.1 Rotation about a fixed axis through $G$

4.3.2 Rotation about a fixed axis not through $G$

4.4 Moments of Inertia and Radius of Gyration

4.4.1 Uniform thin ring

4.4.2 Uniform disc

4.4.3 Hollow disc

4.4.4 Polar moment of inertia of non-circular lamina

4.4.5 Radius of gyration

4.5 Examples

4.5.1 Flywheel with friction torque

4.5.2 Disc rotating about an axis through its periphery

4.5.3 Wheel-and-axle lifting machine
3. Develops and uses the simple theory of torsion of circularsection bars

Describes the assumptions necessary to develop the simple theory of torsion of circular-section bars.

Derives from first principles the relationship between shear strain and twist per unit length, i.e.

$$
\gamma=\frac{r \theta}{l}=\frac{\tau}{G}
$$

Uses the equation of equilibrium to derive the further relationship

$$
T=\frac{G J \theta}{l}
$$

Solves problems involving torsion in solid and hollow shafts.

B. DYNAMICS

4. States the laws of, and solves problems on, angular motion States the equations of motion, for angular motion with constant angular acceleration.

Solves problems involving constant angular acceleration.

Derives from first principles the relationship between applied torque, angular acceleration and moment of inertia.

Defines radius of gyration, $k$, by reference to the expression $I$ $=m k^{2}$.

Solves problems relating to the angular motion of discs and flywheels.

Derives from first principles the expression $r \omega^{2}$ for centripetal acceleration of a body moving in a circle with uniform angular velocity.

Solves problems involving motion of bodies in a circle including considerations of banking. 
4.5.4 Cyclist rounding a curve

4.5.5 Vehicle on a banked track

66

5 Simple Harmonic Motion

5.1 Harmonic Motion

5.2 Coil-spring-Mass System

5.3 Single-spring-Mass System

5.4 Initial Conditions

5.5 Phasor Representation

5.6 Forcing Frequency

5.7 Simple Pendulum

5.8 Problems

6 Kinetic Energy

6.1 Linear Kinetic Energy

6.2 Rotational Kinetic Energy

6.3 Potential and Kinetic Energy

6.4.2 Wheel and axle

6.4.3 Motor lift system

6.5 Momentum

6.5.1 Linear momentum

6.5.2 Moment of momentum (angular momentum)

6.6 Problems involving Energy and Momentum

6.6.1 Collision of railway trucks

6.6.2 Disc clutch

\section{PART THREE FLUIDS IN MOTION}

7 Flow of Fluids

7.1 Mechanics of Steady Flow

7.2 Bernoulli's Equation for the Steady Flow of an Incompressible Fluid
6.4 Examples

6.4.1 Man pushing trolley
5. Describes, and solves problems involving, simple harmonic motion

Describes the relationships between (a) restoring force and displacement (b) displacement and time (c) velocity and time (d) acceleration and displacement.

Defines simple harmonic motion.

Derives the interrelationships between the quantities specified in (a), (b), (c) and (d) above.

Relates simple harmonic motion to circular motion of a phasor and circular frequency.

Solves problems involving simple harmonic motion including the simple pendulum and body supported by a spring.

Describes resonance as occuring when the applied frequency equals the natural frequency.

Discuss the problems that can arise when resonance occurs, e.g. in the use of tools.

6. Describes, and solves problems, involving linear and angular kinetic energy

Derives from first principles the expressions for (a) linear kinetic energy and (b) angular kinetic energy of a body.

Solves problems involving linear and/or angular kinetic energy, including flywheels and lift systems.

\section{FLUIDS IN MOTION}

States Bernoulli's equation.

States the equation of continuity for steady flow through tapered pipes.
7. States and uses Bernoulli's equation 
7.3 Mass-flow-Continuity Equations

7.4 Flow from Tanks

7.5 Flow in a Converging Tube

7.5.1 The venturi meter

7.5.2 Inclined pipe system

7.6 Orifices

7.6.1 Discharge from one tank to another through an orifice

7.6.2 Time to empty a tank

7.7 Impact of a Jet of Fluid on to a Flat Plate

7.8 Problems
108

108

109

110

111

114

115

115

117

119 flow of liquids through pipes, including tapered and inclined pipes and orifices.

Uses the momentum principle to calculate the force produced by the impact of a liquid jet on a normal flat plate. 


\section{Preface}

This book follows the Standard TEC Unit Mechanical Science III which is a third-level Unit in the certificate programmes in mechanical and production engineering.

The specification of the course is as follows.

\section{Unit Title \\ Unit Level \\ Unit Value \\ Design Length \\ Prerequisite Units \\ Credits for Units \\ Aims of Unit}

Special Note

\author{
Mechanical Science \\ III \\ One \\ 60 hours \\ TEC U75/036 Engineering \\ Science II \\ None
}

To develop the student's analytical techniques in the application of scientific principles to mechanical engineering situations

The Unit is designed to be studied concurrently with or after TEC U75/037 Engineering Science III.

To fulfil the aims of the Unit, the book is written with reference to Engineering Science II and recognises the fact that Engineering Science III may be studied concurrently. However, it is assumed that only the statics and dynamics sections of Engineering Science II are needed as far as Mechanical Science III is concerned, although some small overlap between the two is inevitable and consequently forms some revision of essential information.

The combination of Physical Science I and Engineering Science II collectively covers forces on materials, dynamics, static equilibrium of forces and pressure in fluids in sufficient detail to be able to cover the contents of Mechanical Science III (and the relevant section of mechanics in Engineering Science III if this is studied concurrently).

The contents of Mechanical Science III are

(1) Stress, Strain and Elasticity of Materials

(2) Simple Theory of Bending of Symmetrical Beams

(3) Simple Theory of Torsion of Circular Section Bars

(4) Laws of Angular Motion 
(5) Simple Harmonic Motion

(6) Linear and Angular Kinetic Energy

(7) The Application of Bernoulli's Equation to Fluids in Motion.

These topics are covered essentially from first principles using these to show where any formula, which is to be applied to problems, comes from. The range of problems covered goes slightly beyond the bare minimum required, so as to allow the student an opportunity of extending his mechanical engineering knowledge for the purpose of progression in the fields of mechanical and production engineering.

Our thanks go to Mrs P. R. Lancaster for the careful typing of the script and to Dr P. Gallagher of Bradford College for his help in the early stages of preparation.

March 1977

P. R. LANCASTER

D. MITCHELL 